\title{
Intermédialités
}

Histoire et théorie des arts, des lettres et des techniques

Intermediality

History and Theory of the Arts, Literature and Technologies

\section{Intermédialité : ressemblances de famille}

\section{Éric Méchoulan}

Numéro 16, automne 2010

URI : https://id.erudit.org/iderudit/1001965ar

DOI : https://doi.org/10.7202/1001965ar

Aller au sommaire du numéro

Éditeur(s)

Revue intermédialités (Presses de l’Université de Montréal)

ISSN

1705-8546 (imprimé)

1920-3136 (numérique)

Découvrir la revue

Citer cet article

Méchoulan, É. (2010). Intermédialité : ressemblances de famille. Intermédialités / Intermediality, (16), 233-259. https://doi.org/10.7202/1001965ar
Résumé de l'article

Dans cet article, l'auteur explore les différentes conceptualisations proches des théories de l'intermédialité depuis les anciennes pratiques de l'ekphrasis jusqu'aux récentes conceptions de la médiologie en les regroupant sous des genres d'opération différents : parler, voir, communiquer, agir, relier. Il en souligne les proximités et surtout insiste sur le fait que ces théorisations mettent l'accent sur les relations plus que sur les substances. 


\title{
Intermédialité : ressemblances de famille
}

\author{
Éric MÉchoulan
}

a notion d'intermédialité est en partie le résultat d'interactions avec des - ensembles théoriques et avec des pratiques critiques qui, à des titres divers, mettent de l'avant les relations plus que les substances. La notion de «ressemblance de famille» est utilisée par Ludwig Wittgenstein pour éviter le caractère immédiatement référentiel ou essentiel d'un concept et pour souligner que chaque interprétation conceptuelle nécessite la prise en compte d'un contexte dans lequel ce sont ses différentes applications, ses diverses expérimentations, au sein d'un certain jeu de langage, qui donnent au concept des significations offrant certains traits de ressemblance ${ }^{1}$. On y sent bien là un refus des essences $a$ priori et des définitions toutes faites.

Les recherches intermédiales en reprennent volontiers le principe, à condition d'écarter un strict modèle génétique qui verrait dans ces ressemblances de famille conceptuelle des transmissions directes, des influences évidentes et des reprises globales. L'intermédialité problématise justement ces illusions familiales et nous oblige à repenser l'idée même de ressemblances de famille et d'héritage à partir de conceptions qui évitent une simple linéarité de l'histoire ou une affaire de pères et de fils. D'où ces pas de côté théoriques où les analogies, même bancales, semblent plus riches et instructives que les causalités monomaniaques. Plutôt que de généalogie au sens strict, on s'occupera donc de généanalogie.

Le paradigme technique est généralement celui qui semble «naturellement» s'imposer lorsqu'on imagine parler des médias et, à partir de là, de l'intermédialité: de l'arraisonnement dramatique du monde moderne par la technique, ainsi que l'envisage Martin Heidegger, au pétillement ironique du mot de Marshall

1. Ludwig Wittgenstein, Recherches philosophiques [1953], trad. Françoise Dastur, Maurice Élie, Jean-Luc Gautero, Dominique Janicaud et Élisabeth Rigal, préf. Élisabeth Rigal, Paris, Gallimard, coll. «Bibliothèque de philosophie », 2004. 
McLuhan «le medium est le message», on pourrait décliner les différentes positions qui allouent, aux instruments et aux moyens, la posture de fins ou de résultats. Leur importance est notable, surtout quand elles permettent les nuances et qu'elles signalent des contraintes ou des productions, sans tomber dans le piège du causalisme rigide ou du déterminisme aveugle. Ainsi, ce que Marcel Mauss analyse comme «techniques du corps ${ }^{2}$ » (la façon dont les gestes apparemment les plus naturels: marcher, se tenir assis, manger, sont assimilés culturellement et ressortissent donc d'intériorisations de modes comportementaux) ou Michel Foucault comme «techniques de soi $^{3}$ » (processus de subjectivation dans lesquels les êtres se construisent comme individus sociaux) indiquent que la notion même de technique ici ne concerne plus simplement l'outillage manuel inventé par les hommes, mais surtout «l'outillage mental» (pour reprendre le terme de l'historien Lucien Febvre).

«Technique » devient alors presque une métonymie pour «social» : c'est bien ce qui lui donne son allure «naturelle» tant elle colle à la peau de nos rôles sociaux. Réciproquement, les conceptions de la technique soulignent volontiers qu'elle est elle-même le "produit d'un système social spécifique ${ }^{4}$ », comme le disait Raymond Williams. Lorsqu'on en vient à glisser sous les opérations techniques le développement des «médias", on en arrive à la conception dominante aujourd'hui d'une équivalence entre l'imprégnation en chaque être des techniques mobilisées, la mise en forme des individus par leurs cultures et leur immersion dans les multiples modes de communication générés par les médias. Tout cela est très juste.

Cependant, il est des types d'analyses, parfois très anciennes, qui s'avèrent aussi intéressantes, dans la mesure où l'affairement technique y est considéré surtout à partir des effets des relations. Cela évite, du coup, ce faux effet de naturalité qui consiste à mettre automatiquement les techniques au cœur des opérations intermédiales. Nous pouvons en décliner quelques figures sous cinq genres voisins d'opération qui se recoupent et interfèrent: parler, voir, communiquer, agir, relier. Autant de déclinaisons qui, en évitant toute mise en rapport

2. Marcel Mauss, «Les techniques du corps», Journal de psychologie, vol. 32, $\mathrm{n}^{\circ}$ 3-4, 1936, repris dans Sociologie et anthropologie, Paris, Les Presses universitaires de France, coll. «Bibliothèque de sociologie contemporaine », 1950, p. 363-386.

3. Michel Foucault, «Les techniques de soi », Dits et écrits, IV, Paris, Gallimard, coll. «Bibliothèque des sciences humaines», 1994, p. 783-812.

4. Raymond Williams, Television: Technology and Cultural Form, Londres, Fontana, 1974, p. 135. 
trop singulière, ne visent pourtant pas à l'exhaustivités. Du point de vue du savoir, l'avantage réside dans la succession rapide de juxtapositions (que l'on espère) éclairantes, plutôt que dans l'exploration scrupuleuse de telle ou telle comparaison - la rapidité de ces résumés montrant au moins l'intérêt du tempo pour la pensée et l'utilité du rythme pour l'intermédialité.

\section{PARLER}

Le dialogisme - Mikhaïl Bakhtine est un poéticien russe qui tente d'analyser les rapports esthétiques et sociaux à même l'expérience du langage. Il insiste en effet sur le fait qu'une parole est toujours adressée à quelqu'un dans un certain contexte d'énonciation. On ne parle jamais à une personne dont le cerveau serait miraculeusement vide de mots et d'expressions; notre message entre en réalité en interaction avec tout un milieu de compréhension constitué par l'histoire d'un individu avec ses savoirs sédimentés et ses habitudes langagières. Le sujet linguistique n'est jamais clos sur lui-même; il est le produit de ses interactions, au besoin polémiques, avec les autres. C'est pourquoi Bakhtine, avec le dialogisme, oriente la linguistique et la poétique vers les usages de la langue, donc vers les utilisations et les utilisateurs : tout message y apparaît, non pas neutre et pur, mais diffracté ou réfracté dans la conscience du récepteur. Bakhtine insiste à chaque fois sur les contextes d'énonciation dans lesquels les usages connus prennent vie. À l'inverse d'un Saussure, père de la linguistique moderne, qui divise le langage entre langue (compétence générale) et parole (performance actuelle) pour mieux ramener l'ordre de la science linguistique à la seule régularité de la langue, Bakhtine essaye d'en saisir l'entrelacement: c'est dans les opérations de la parole, plus encore dans les mouvements même du dialogue, que la langue, avec ses règles, apparaît.

Ceci l'amène à étudier les «genres » du discours. Les usages supposent en effet des possibilités de reconnaissance des types d'énoncés: on ne produit pas de la même façon un discours politique, un sonnet romantique, un mode d'emploi de machine à laver ou un roman policier. Le propre des textes littéraires véritablement dialogiques consiste justement en l'étroit entremêlement de ces genres et de la multiplicité des usages quotidiens. Pour Bakhtine, la culture n'est pas constituée par un territoire bien connu et des frontières stables, mais faite de

5. Ce texte constitue la version longue d'un chapitre prévu pour un «Que sais-je?» sur l'intermédialité, d’où son caractère plutôt didactique. 
multiples frontières que l'on suit ou que l'on traverse sans cesse ${ }^{6}$. Ces frontières n'enferment pas un ensemble homogène, elles forment le maillage variable historiquement de genres de discours hétérogènes qui se trouvent ainsi configurés.

Dans ses derniers textes, Bakhtine met de l'avant deux notions: exotopie et compréhension. L'exotopie (vnenakhodimost', littéralement: «le fait de se trouver au dehors») suppose que l'on ne se contente pas de recevoir des messages, mais que l'on joue aussi sur la capacité de chacun à imaginer les points de vue étrangers aux siens, afin de mieux combiner et associer les perspectives et assurer la compréhension. C'est là le deuxième terme important, dont on peut apprécier la valeur du préfixe «cum » (avec): comprendre, c'est parler et penser avec les autres (ce qui veut aussi dire contre les autres). L'anthropologie bakhtinienne repose sur une ontologie implicite: non focalisée sur l'être comme tel ou sur la conscience du sujet, mais sur l'être-avec, autrement dit sur un être de langage immédiatement plongé dans les médiations sociales.

L'intertextualité - Reprenant les théories de Bakhtine, certains poéticiens des années 1960-1970 introduisent la notion d'intertextualité. Au moment où les analyses se focalisent volontiers sur le Texte, cela permet de réintroduire une certaine historicité et une dynamique des interactions. Julia Kristeva en particulier conçoit le texte comme une «mosaïque de textes ${ }^{7} »$. Il ne s'agit pas simplement de reprendre les théories modernistes de l'influence ou des sources, qui réduisent le texte à une reproduction de l'ancien. On serait en fait plus proche des théories classiques de l'imitation et de la variante, qui font de chaque texte une instance singulière d'une longue tradition. Pourtant, l'intertextualité ne se situe pas nécessairement dans la reprise consciente de textes canoniques ou dans la variation jouée d'usages bien connus. Elle constitue plutôt des formes de recyclage conscient ou non d'autres textes; elle impose de penser d'abord un réseau de textes, voire une bibliothèque ou une mémoire d'œuvres, plutôt que de partir d'un ouvrage pour y convoquer d'autres textes.

Ainsi, l'intertextualité sort le texte de son autonomie supposée et permet de lire en lui la mise en œuvre d'autres textes préexistants, par transposition, travestissement, parodie, citation, allusion, etc. ${ }^{8}$. L'intextualité repositionne alors le texte considéré dans une chaîne d'énoncés et mesure son rapport à des œuvres

6. Mikhail Bakhtine, Esthétique et théorie du roman [1975], trad. Daria Olivier, préf. Michel Aucouturier, Paris, Gallimard, coll. «Bibliothèque des idées», 1978, p. 40.

7. Julia Kristeva, Semeiotikè: recherches pour une sémanalyse, Paris, Éditions du Seuil, coll. «Tel quel », 1969, p. 85 .

8. Pour une typologie possible, voir Gérard Genette, Palimpsestes. La littérature au second degré, Paris, Éditions du Seuil, coll. «Poétique », 1982. 
antérieures - voire postérieures: plagiat par anticipation, comme Raymond Roussel et certaines pratiques de l'OULIPO, ou reprise modifiant la perception et l'interprétation d'ouvrages préexistants, comme lorsque Bacon repeint le portrait du pape Innocent X de Velazquez (1953) ou que Jean-Luc Godard, recyclant à sa manière l'opéra de Bizet, filme Prénom Carmen (1983), donnant ainsi une profondeur anachronique (nous reviendrons sur cette notion) aux œuvres de Velazquez et de Bizet? .

L'interdiscursivité - Toutes ces enquêtes «intertextuelles» demeurent pourtant dans l'homogénéité d'une tradition. Elles supposent, non l'autonomie des œuvres, mais l'autonomie d'un champ de production esthétique (littéraire, pictural ou musical), ou bien du champ artistique en général par rapport à toutes les autres productions sociales. Or, l'ordre des œuvres ne dépend pas seulement de la sphère institutionnelle à laquelle elles appartiennent, mais aussi des multiples «discours » et «représentations » qui s’y trouvent ramassés, tressés, traversés: discours quotidiens, discours des disciplines ou des champs de compétence (c'est déjà ce que le dialogisme bakhtinien impliquait). Chaque ouvrage suppose alors la performance de diverses compétences dont doit rendre compte une «interdiscursivité».

Ainsi, Pierrot le fou (1965) de Jean-Luc Godard intègre ouvertement le discours publicitaire comme celui de l'histoire de l'art: la gaine Confort et les pages d'Élie Faure sur Velazquez se mêlent à l'histoire des guerres coloniales et des aventures romanesques des deux amants. Du côté littéraire, on peut voir Flaubert entrelacer, dans la scène des comices agricoles de Madame Bovary (1857), les paroles amoureuses d'Emma et Rodolphe aux discours d'ouverture pontifiants des autorités, opposant et reliant l'intensité passionnée des sentiments à la bêtise orchestrée des hommes d'institution. L'interdiscursivité suppose une multiplicité de discours dont les productions artistiques sont en fait tissées.

Plus largement encore, chaque œuvre mobilise et s'inscrit dans des formations discursives qui articulent le représentable à des représentés historiquement circonscrits. Michel Foucault décrit les formations discursives comme des règles de transformation des énoncés. Au lieu de voir un sujet, parfaitement conscient, organiser ce qu'il dit dans un langage assez transparent pour lier des pensées à des choses, l'insistance sur l'opacité du langage amène Foucault à privilégier l'analyse scrupuleuse des situations de discours et des règles qui permettent l'utilisation

9. Pour ce renversement de perspective chronologique, du côté de l'histoire de l'art, voir Michael Baxandall, Patterns of Intention: On the Historical Explanation of Pictures, New Haven, Yale University Press, 1986, p. 58-62. 
et la compréhension des pratiques discursives ${ }^{10}$. En Allemagne, Jürgen Link et Ursula Link-Heer réarticulent cette analyse de discours en examinant les symboles collectifs et en prenant en compte les recherches iconographiques dans l'analyse des textes ${ }^{11}$. Il faut donc saisir aussi l'importance des images au sein même de ce que l'on appelle ainsi «interdiscours». Par «texte», on peut en effet entendre toute production esthétique qui constitue un «tissu» de mots, de sons, de pigments ou d'images: ainsi, l'iconologie panofskienne, dès les années 1930, est, bien avant les intertextualités mises à la mode par la critique littéraire, la recherche de motifs ou de dispositifs iconiques qui essaiment de toile en toile, ouvrant ainsi sur une «intericonicité».

VOIR

L'intericonicité: transferts et transmission - Quand Erwin Panofsky rédige un ouvrage où il met en parallèle la construction des cathédrales gothiques et l'architecture de la théologie scolastique ${ }^{12}$, il tâche de saisir par cette mise en rapport surprenante non seulement des similitudes intellectuelles caractéristiques d'une époque, mais une relation de cause à effet: les habitudes de division intellectuelle du raisonnement dans la scolastique produiraient une division formelle rigoureuse des cathédrales, puisque leur plan redonne à l'échelle réduite de chacun des éléments les proportions de l'ensemble. Autant ce régime intellectuel des proportions permet, en effet, de passer d'un domaine (la théologie) à un autre (l'architecture), autant la «clarté», dont Panofsky fait aussi un effet de la scolastique sur la construction des cathédrales, est plus sujette à caution. En même temps, il développe une science qu'il appelle «iconologie», dont le but est de repérer les motifs récurrents dans les œuvres et d'en suivre les variations historiques, grâce à un examen à trois niveaux: expérience de reconnaissances de formes familières (par exemple, un homme muni d'un couteau et un enfant); connaissance des sources écrites (ces personnages peuvent représenter Abraham sur le point de sacrifier son fils Isaac); intuition des valeurs symboliques (soumission à Dieu, préfiguration du sacrifice du Christ). Panofsky s'intéresse donc aux transferts possibles entre images, textes et valeurs symboliques d'une société.

10. Michel Foucault, L'archéologie du savoir, Paris, Gallimard, coll. «Bibliothèque des sciences humaines", 1969, en particulier p. 65-67.

11. Jürgen Link, «Kollektivsymbole und Mediendiskurse », kultuRRevolution, $\mathrm{n}^{\circ}$ 1, 1982 , p. 6-21.

12. Erwin Panofsky, Architecture gothique et pensée scolastique [1951], trad. Pierre Bourdieu, Paris, Les Éditions de Minuit, coll. «Le sens commun », 1967. 
Son maître, Aby Warburg, a eu moins d'influence sur le moment, mais son œuvre est revenue en force sur la scène intellectuelle depuis les années 1980. Autant le premier vise la pureté d'une signification que la recherche historique permettrait d'exhiber, autant le second est en quête des petites impuretés de temps qui permettent aux images fossilisées de reprendre vie dans la mémoire des civilisations. Contre le formalisme esthétique, Aby Warburg ramène, en effet, obstinément les images à leur puissance mémorielle. Fasciné par la survie de l'Antiquité dans l'art de la Renaissance ou par la rémanence de pratiques rituelles indiennes quand il visite les États-Unis en 1896, il consacre toute sa vie à la question de la transmission, jusque dans ses manques flagrants ou ses caractères inconscients. Du même coup, il propose une anthropologie historique des images ou une «histoire iconologique des civilisations» qui dépassent l'intericonicité suggérée par Panofsky ou Focillon, qui insistent plutôt sur une certaine «vie des formes ${ }^{13} »$ : chez Warburg, pour comprendre les images, il faut reconstituer des contextes mémoriels où elles prennent forme, sens, vie; et pour saisir les cultures, il faut suivre les transformations, détournements, reprises des images au fil des époques. Cette histoire de l'art est une « iconologie de l'intervalle», écrit-il en 1929, ou plutôt de «l'espace-entre ", Zwischenraum, sachant que cet «espaceentre» ne désigne rien de vide, aucun «no man's land» comme on l'a parfois pensé, mais un espace de temps, une durée mémorielle. La recherche porte justement sur ces glissements entre les êtres, les signes, les moments, les images, par lesquels se construisent des médiations symboliques et des formes de vie sans cesse renaissantes ou survivantes. C'est pourquoi Warburg ouvre l'histoire de l'art à des objets visuels qui n'en faisaient pas partie faute de «dignité esthétique »: gravures, photographies, etc. ${ }^{14}$.

Le meilleur exemple de ce travail sur les transmissions se trouve sans doute dans la bibliothèque qu'il a lentement édifiée, placée sous le nom de Mnemosyne (la déesse grecque de la mémoire, mère des muses): son classement, étrange aux yeux des bibliothécaires professionnels, répond en fait à la logique d'une recherche (variant donc à mesure de celle-ci). Son dernier état, conservé (mais

13. En effet, pour l'historien de l'art, Henri Focillon, il y a une continuité entre vie, nature et art du point de vue des «relations formelles dans une œuvre et entre les œuvres», car «la vie est forme, et la forme le mode de la vie». Henri Focillon, Vie des formes [1934], Paris, Les Presses universitaires de France, coll. «Quadrige», 1970, p. 3.

14. Aby Warburg, Essais florentins, trad. Sibylle Müller, Paris, Klincksieck, 1990; Le rituel du serpent. Art et anthropologie, introduction de Joseph L. Koener, Paris, Macula, 2003. Voir Karl Sierek, Images oiseaux: Aby Warburg et la théorie des médias, trad. Pierre Rusch, Paris, Klincksieck, 2009. 
aussi modifié et enrichi) à Londres, comprend quatre sections générales: image (histoire de l'art, archéologie), mot (littératures, transmission des textes), orientation (religions occidentale et orientale et philosophie), action (histoire sociale, politique, magie et science). Ces catégories associent les livres et font de la bibliothèque elle-même un véritable média: un lieu d'organisation de la pensée et de transmission des données. En arrangeant par thèmes proches les uns des autres des ensembles d'ouvrages, il souscrit à la théorie du «bon voisinage » qui suppose que la réponse à ses problèmes réside moins dans le livre que l’on cherche que dans celui d'à côté. Si l'histoire déplace sans cesse ses objets comme un fleuve ses petits cailloux, alors le trajet de l'historien, pour Warburg, ne peut suivre de lignes droites. Ce sont les impuretés de l'histoire qui permettent la cristallisation des interprétations.

Ekphrasis: texte et image - Panofsky et Warburg associaient dans leurs recherches écrits et images. Or, depuis l'Antiquité, une technique lie texte et peinture par l'expressivité de l'ekphrasis: «L'ekphrasis est un discours détaillé; grâce à ce que l'on appelle sa "vivacité visuelle" (enargeia), il met sous les yeux ce qu'il montre ${ }^{15}$.» Il n'y a pas ici simple déplacement de l'argumentatif vers le narratif, mais surtout reprise d'un exercice topique pour définir un lieu commun. Lusage le plus ancien en est la description que fait Homère du bouclier d'Achille et des multiples figures qui le composent. Même si l'ekphrasis peut servir à montrer n'importe quel paysage ou décor, la tradition valorise en fait la description d'œuvres d'art, redoublant ainsi la valeur littéraire par la valeur picturale ou architecturale.

À partir des ouvrages littéraires, le genre devient presque autonome, s'insérant comme un morceau de bravoure dans les textes (Lucien en particulier en fait un usage important) ou prenant la forme de recueils spécialisés dans ces portraits (Eikones, « icônes », pour reprendre le titre donné au plus ancien recueil d'ekphraseis que nous connaissions, celui de Philostrate le rhéteur). Les ekphraseis circulent alors comme des morceaux d'anthologie, alimentant l'usage de lieux communs de la tradition (des topoï), qui renvoient à l'espace d'une communauté ainsi tressée par ces lieux communs (et non aux «clichés» de la modernité: un cliché est un lieu commun dont la valeur commune s'est desséchée). Ainsi, des compétences linguistiques permettent de faire voir immédiatement ce que le lecteur ou l'auditeur n'a pas sous les yeux. Important pour les citoyens ou les

15. Hermogène de Tarse, cité par Michael Baxandall, Les Humanistes à la découverte de la composition en peinture, 1340-1450, trad. Maurice Brock, Paris, Éditions du Seuil, coll. «Des travaux », 1989, p. 111. 
avocats qui cherchent à emporter l'adhésion du public, l'ekphrasis glisse vers les exercices scolaires de la rhétorique ou les morceaux de bravoure poétiques: «cette unité (paysage, portrait) quitte le discours oratoire (juridique, politique) et s'intègre facilement dans la narration, dans le continu romanesque: une fois de plus, la rhétorique "mord" sur le littéraire ${ }^{16}$ ».

En fait, il faut prendre au sérieux cette image proposée par Roland Barthes. Le propre de l'ekphrasis est d'empiéter sur des frontières, de troubler les séparations apparemment évidentes entre le visible et le lisible, de donner au médium de l'écriture l'énergie de la vue. De manière générale, on peut voir ainsi que la catégorie d'enargeia permet de traverser les domaines des sens et des pratiques institutionnalisées. Ezra Pound retrouve cette puissance de l'enargeia dans ce qu'il appelle, lui, phanopoia, qui lui permet de penser la force de l'image:

An «Image» is that which presents an intellectual and emotional complex in an instant of time. [...] It is the presentation of such a «complex» instantaneously which gives that sense of sudden liberation [...]; that sense of sudden growth, which we experience in the presence of the greatest works of art ${ }^{17}$.

On saisit ainsi, de l'Antiquité aux temps modernes, que l'ekphrasis est autant une affaire de temps que d'espace, autant une question d'idée que d'image. De même que l'écrit ouvre sur du visible, la description contracte la durée dans l'instantanéité de la vision pour mieux lui donner l'intensité du temps.

Si Cicéron tâche, dans sa traduction du terme grec enargeia, de distinguer le travail de la logique philosophique de l'evidentia et le travail de l'imagination rhétorique du sub oculos subjectio («mettre sous les yeux»), Quintilien n’hésite pas à les réunir sous la même «évidence »: l'instantané de la sensation de présence emporte avec elle l'intuition rationnelle d'une vérité. Parce qu'elle mettrait sous les yeux grâce aux mots, l'ekphrasis lierait présence et vérité. Il est d'autant plus ironique de noter que le recueil des Eikones de Philostrate constituait soit la description littéraire de fresques réelles, soit l'invention écrite de peintures fictives: rien ne permet de le savoir et cela n'importe guère. L'écrit lie (lit?) visible et véritable; il en fabrique la relation effective.

16. Roland Barthes, «L'ancienne rhétorique: aide-mémoire», repris dans Euvres complètes, tome 2: «1966-1973», Paris, Éditions du Seuil, 1994, p. 913.

17. Ezra Pound, «A Retrospect» (1918), repris dans Vassiliki Kolocotroni, Jane Goldman, Olga Taxidou (dir.), Modernism: An Anthology of Sources and Documents, Chicago, The University of Chicago Press, 1998, p. 374. Voir Benoit Turquety, «L'imagearrêt. Pound, Zudofsky, Mallarmé, Huillet et Straub : poésie et cinéma », www.fabula.org/ lht/2/Turquety.html (dernière consultation le 17 janvier 2011). 
Lallégorèse : texte, image et idée - C'est encore une vérité devenue problématique que le processus de l'allégorèse a pour tâche de retrouver. Là où les «mâ̂tres de vérité ${ }^{18}$ » anciens, ceux que nous considérons encore comme des «poètes » ou des «sages", offraient à leurs communautés une parole sûre, la mise par écrit de leurs propos, entre le $8^{\mathrm{e}}$ et le $6^{\mathrm{e}}$ siècle avant Jésus-Christ, en fige les éléments et en date irrémédiablement les énoncés. Or, ce changement de médium (de la tradition orale, adaptant les paroles aux situations d'énonciation, à l'écriture qui en fixe les éléments et en ressasse l'autorité) génère une gêne évidente avec le temps qui passe: la langue évolue et rend impertinents ou incompréhensibles certains énoncés, les mœurs changent et donnent à d'anciennes vertus l'apparence de vices de forme. Ainsi, les discours choquants sur les dieux tenus par Homère ou Hésiode doivent être réinterprétés. Dans la mesure où ces textes ont été «sacralisés», ils ne peuvent tenir des discours inconvenants: on suppose alors qu'ils ne disent pas exactement ce qu'ils énoncent, qu'ils voilent même sous des images absurdes ou inopportunes les principes mêmes par lesquels on voudrait en condamner les effets. Côté production, l'allégorie, cette manière autre (allos) de parler en public (agoreuein), permet ainsi de glisser sous des apparences surprenantes une parole véridique; côté réception, l'allégorèse est le processus d'interprétation qui repère et dévoile cette vérité sous les aspects incongrus et les opinions irrecevables ${ }^{19}$.

Par la suite, les stoïciens s'en servent pour proposer deux expressions de la vérité, deux niveaux d'une même réalité: le mythe pour le poète, la raison pour le philosophe. La théologie chrétienne lie l'allégorie à la parabole biblique ou à la typologie (un personnage ou un événement de la Bible en préfigure un autre, révélant ainsi le plan divin du salut des hommes ${ }^{20}$ ) et autorise des effets de sens en jouant sur les différents niveaux de la référence littérale ou de la signification allégorique. De manière plus large, on peut voir que l'allégorie est très utile, dans un univers social et cosmique hiérarchisé, pour passer d'un niveau à un autre: ainsi, pour Avicenne, l'ascension sacrée du prophète Mohammed est décrite dans les termes rationalistes d'une théorie de la connaissance ${ }^{21}$. Lorsque

18. Voir Marcel Detienne, Les maitres de vérité dans la Grèce archaïque, préf. Pierre Vidal-Naquet, Paris, La Découverte, 1967.

19. Jean Pépin, La tradition de l'allégorie de Philon d'Alexandrie à Dante, Paris, Études augustiniennes, 1987.

20. Armand Strubel, "Allegoria in factis" et "allegoria in verbis" ", Poétique, n 23, 1975, p. 350-365.

21. Peter Heath, Allegory and Philosophy in Avicenna (Ibn Sînâ), Philadelphie, University of Pennsylvania Press, 1992. 
ce monde hiérarchisé et fini cède la place à un univers géométrique homogène infini et lorsque les mythes des «sauvages» découverts en Amérique semblent à la fois irrationnels et proches des mythes grecs, l'allégorie est réduite à un code d'écriture, à une figure stylistique (la personnification) et elle ne fonctionne plus comme un opérateur de passage entre deux niveaux de réalité, deux vecteurs de vérité: le monde de l'allégorie ou du mythe apparaît comme une préhistoire de la raison humaine que la philosophie et la science ont dépassé. C'est pourquoi les romantiques dévaluent l'allégorie au profit du symbole qui aurait gardé, lui, un caractère organique.

Or, c'est dans ce contexte que l'on voit apparaître pour la première fois le terme de «intermedium » : à propos de Spenser, ce grand poète de la Renaissance, Coleridge souligne que «narrative allegory is distinguished from mythology as reality from symbol; it is in short the proper intermedium between person and personification ${ }^{22}$ ». Autrement dit, c'est précisément le caractère «intermédial» de l'allégorie qui l'intéresse, son déplacement entre la réalité d'une personne physique et la représentation imaginaire d'une idée, bref la relation entre sensible et intelligible. C'est ce qui explique la valeur qu'il accorde, pour une fois, à l'allégorie aux dépens du symbole, car celui-ci peut tourner trop vite à l'identité, alors que celle-là, jusque dans son aspect conventionnel, permet de lier les opposés tout en maintenant à distance la violence du littéral23.

On comprend alors l'intérêt que peuvent y trouver Walter Benjamin ou Paul de Man: l'allégorie relève d'une philosophie de la différence, d'une pensée de l'origine et de l'écart. Pour Walter Benjamin, l'allégorie dans les drames baroques du $17^{\mathrm{e}}$ siècle est à la pensée ce que les ruines sont aux choses du monde, un processus qui est fantôme présent d'un passé disparu. Lallégorie est l'expérience que l'on peut faire du monde une fois que celui-ci est perçu comme contingent et fragmenté: cette fragmentation a sa vertu, sa dynamique propre, celle de la nécessité des relations. Mais le retour de l'allégorie chez Baudelaire consonne avec le fétichisme de la marchandise analysé par Marx et permet d'en figurer la logique sociale: l'aliénation des êtres dans le monde des marchandises est une fragmentation de l'expérience ${ }^{24}$.

22. Samuel Taylor Coleridge, Literary Remains, vol. 1, 2003, infomotions.com/ etexts/gutenberg/dirs/etexto5/7remı.htm (dernière consultation le 6 janvier 2011).

23. Voir Steven Knapp, Personification and the Sublime, Cambridge (Mass.) et Londres, Harvard University Press, 1985, p. 22-28.

24. Voir Walter Benjamin, Origine du drame baroque allemand, trad. Sibylle Müller, André Hirt, Irving Wohlfarth, Paris, Flammarion, coll. «La philosophie en effet», 1985, et 
Paul de Man radicalise le rapport entre allégorie et lecture en faisant de celle-là la constante reprise de l'impossibilité de celle-ci, d'où l'intérêt de l'allégorie qui, à l'inverse du symbole, facile procureur d'identification, désigne avant tout une distance en relation avec sa propre origine:

It remains necessary, if there is to be allegory, that the allegorical sign refers to another sign that precedes it. [...] Whereas the symbol postulates the possibility of an identity or identification, allegory designates primarily a distance in relation to its own origin ${ }^{25}$.

L'allégorie est à la fois différence et relation, écart et lien.

\section{COMMUNIQUER}

244 Malgré cela, l'interdiscursivité ou l'intericonicité demeurent souvent implicitement ancrées dans des opérations langagières ou visuelles comme si les paroles étaient indépendantes des effets sociaux de l'oralité et de la singularité physique des performances, comme si les écrits demeuraient étrangers à la matérialité des dispositifs d'impression et de diffusion et aux institutions qui les autorisent, comme si les images étaient indifférentes à leurs techniques de fabrication, de transmission et de réception. Or, la paléontologie d'un Leroi-Gourhan ${ }^{26}$ nous a appris comment la genèse de l'homo sapiens doit prendre en compte des éléments aussi hétérogènes que la station debout (qui libère la bouche de la cueillette de nourriture pour commencer à parler ou embrasser, qui libère la main de la marche pour mieux s'outiller ou caresser) et l'appareil de mémoire collective (qui permet à un être, qui n'est plus génétiquement déterminé, de vivre dans une communauté sans cesse réinventée). De même, l'anthropologie d'un Marcel Mauss s'occupe aussi bien des techniques du corps avec la ritualité des gestes ou des postures (comme nous l'avons vu) que de la construction de réciprocités sociales avec le don et le contre-don ${ }^{27}$. Autant de mises en relation fondamentales entre techniques transmises, usages individuels et formes sociales.

Charles Baudelaire. Un poète lyrique à l'apogée du capitalisme, trad. Jean Lacoste, Paris, Payot \& Rivages, coll. «Petite bibliothèque Payot», 2002.

25. Paul de Man, «Rhetoric of Temporality», Allegories of Reading: Figural Language in Rousseau, Nietzsche, Rilke and Proust, New Haven, Yale University Press, 1979, p. 207.

26. Voir André Leroi-Gourhan, Le geste et la parole, Paris, Albin Michel, 1965.

27. Voir Marcel Mauss, Euvres, Paris, Les Éditions de Minuit, coll. «Le sens commun », 1968-1974. 
Loralité - La communication par la parole nous semble évidente et naturelle. Lêtre humain est, par définition, celui qui mobilise la parole. Il ne faut, pourtant, pas se leurrer sur cette naturalité, car chacun apprend (laborieusement) à s'exprimer en fonction des codes linguistiques. En outre, ces usages de la langue reposent sur des codages sociaux des expressions: la rhétorique, depuis l'Antiquité, ou la sociolinguistique plus récemment permettent de saisir que la langue n'est pas un instrument neutre et transparent.

L'oralité, tout autant que l'écrit, est une technique de communication qui a ses implications, ses avantages et ses inconvénients. Ainsi, l'oralité implique un art de la variante là où l'écrit fige le texte: la tradition non seulement conserve le passé, mais est une force d'animation qui en assure un perpétuel recyclage. L'oralité s'intègre à des sociétés mémorielles, qui développent de nombreux moyens mnémotechniques très efficaces.

Contrairement à ce que nous pourrions croire, ces sociétés sont d'une grande inventivité et d'une grande souplesse dans la réactualisation constante du passé, alors que les sociétés occidentales modernes, régies surtout par l'écrit, ont tendance à figer un passé, devenu de plus en plus étranger, voire énigmatique. Le médiéviste et poéticien Paul Zumthor en saisit toutes les possibilités dans la poésie, que ce soit dans les sociétés traditionnelles ou dans le Moyen Âge occidental, jusque dans la chanson contemporaine ou dans les représentations théâtrales d'avant-garde proches souvent du happening, pour mieux en montrer l'investissement corporel nécessaire dans les rythmes des vers et les enjeux culturels des performances au sein d'une «théâtralité» généralisée ${ }^{28}$.

Cependant, l'écrit n'a pas fait disparaître les pratiques orales, pas plus que le roman n'a remplacé la comédie ou la prose n’a éliminé les vers. Fielding, à une époque où le roman n’a pas encore la légitimité qu'il a acquise depuis le $19^{\mathrm{e}}$ siècle, décrit Tom Jones (1749) comme un "poème épique comique en prose ", mélangeant ainsi les genres et les modes connus de communication. Par ailleurs, que ce soient les chansons ou les rumeurs, ce sont autant d'usages sociaux de l'oralité manifestement encore indispensables au $21^{\mathrm{e}}$ siècle. C'est pourquoi Paul Zumthor propose de différencier oralité sans contact avec l'écrit, oralité mixte (où l'influence de l'écrit demeure marginale) et oralité seconde (dans des sociétés où l'écrit est privilégié). Cela lui permet de saisir de manière nuancée les multiples situations dans lesquelles la parole orale peut séduire, intriguer, appuyer

28. Paul Zumthor, Introduction à la poésie orale, Paris, Éditions du Seuil, coll. «Poétique », 1983, et La lettre et la voix. De la «littérature» médiévale, Paris, Éditions du Seuil, coll. «Poétique», 1987. 
ou déchoir. Ainsi, l'oxymoron «littérature orale» peut devenir historiquement compréhensible et théoriquement déplacé.

Paul Zumthor discerne dès le $12^{\mathrm{e}}$ siècle les premières formes textuelles qui annoncent la «littérature». Mais la voix poétique traditionnelle ne cède qu'avec l'univers tout entier dans lequel elle prenait sens et valeur: «Alors, dans les lointains du $17^{\mathrm{e}}$, du $18^{\mathrm{e}}$ siècle, la littérature aura imprégné le discours poétique d'un besoin de régulation, aura réveillé en lui le désir d'une systématisation des textes. ${ }^{29}$ » Or, il notait auparavant, à propos de ce désir de qui a présidé à l’institution de la «littérature», un élément rarement pris en considération dans nos histoires :

La formation d'un canon, dans la culture d'un groupe social, trahit peut-être l'effroi que ressentent les individus de ce groupe, du fond de leur besoin de survivre. Quelle peur, si cela est vrai, quels doutes, quelle désespérance, a maintenu parmi nous, en leur donnant figure, pendant trois ou quatre siècles, notre «littérature » ${ }^{30}$ ?

Intuition singulière qui lie peur sociale et désir de systématisation, conservation de soi et constitution d'un canon, terreur générale et généralisation des lettres. L'écriture aurait partie liée avec la peur: de fait, elle permet de mettre à distance la présence des autres. Elle relie, certes, mais parce qu'elle éloigne.

L'écriture - Oralité et écriture répondent à des logiques différentes, même si elles s'entrecroisent. Pas plus que l'oralité n'est une technique neutre, l'écriture ne consiste dans la simple répétition de ce que la voix a énoncés1. Prolongeant et problématisant les intuitions de Walter Ong, l'anthropologue Jack Goody va jusqu'à parler d'une « raison graphique » avec un ensemble d'implications sur les formes d'argumentation et les fabrications des mémoires collectives. Reprenant certains éléments mis de l'avant par Marshall McLuhan sur les effets intellectuels et sensoriels des procédures techniques et des moyens de communication ${ }^{32}$, Jack Goody montre comment l'écriture impose un stockage qui fige ce qui est conservé et qui ouvre sur des usages d'analyse logique des énoncés séparés de leurs énonciateurs. La forme même de l'écriture permet une pensée par tableaux systématiques. Ainsi, il relie le développement des usages de l'écrit (manuscrit puis imprimé) à la constitution d'empires bureaucratiques, à l'affirmation sociale

29. Zumthor, 1987, p. 319 .

30. Ibid., p. 314.

31. Voir Walter Jackson Ong, Orality and Literacy: The Technologizing of the Word, Londres, Methuen, 1982.

32. Marshall McLuhan, Understanding Media: The Extensions of Man, New York, McGraw-Hill, 1964. 
d'une élite lettrée et à la valeur accordée aux procédures intellectuelles ${ }^{33}$. Le développement de l'imprimerie depuis le $16^{\mathrm{e}}$ siècle a servi là aussi d'accélérateur pour la constitution des États modernes ${ }^{34}$. Cependant, il ne s'agit pas de processus déterminants de façon rigide, où la technique de communication influerait directement sur les pratiques sociales et intellectuelles. Il faut en fait tenir compte des pratiques d'appropriation singulière de ces usages techniques.

C'est cette notion d'«appropriation » sur laquelle insiste Roger Chartier et, plus largement, les histoires culturelles de l'imprimé. Par exemple, la «culture populaire» ne réside pas dans des corpus tout découpés (par une élite), le «populaire» au contraire "qualifie un mode de relation, une manière d'utiliser des objets ou des normes qui circulent dans toute la société [... caractérisant] les modalités différenciées de leur appropriation ${ }^{35}{ }$. L'histoire de l'imprimé devient ainsi histoire des producteurs, mais aussi des diffuseurs et surtout du public lecteur. Autant de mises en relation dont les historiens établissent les contours et problématisent les variations.

En ce sens, on peut certes insister sur le fait que l'imprimé ne renvoie pas simplement à un appareillage technique, mais à toute une nouvelle culture, sans pour autant en faire une détermination rigide des comportements et des usages. En particulier, l'imprimé ne sonne pas la fin des traditions orales. L'oralité s’adapte à l'écrit et réciproquement, la diffusion imprimée tâche de récupérer les valeurs de l'oral: dialogues, entretiens, conversations permettent de donner une allure de naturel et d'immédiateté même aux traités savants qui les exploitent. Pendant longtemps, les historiens ont vu dans la Révolution française l'effet de la diffusion de l'imprimé et surtout des textes philosophiques. Pourtant, Roger Chartier voit plutôt cette interprétation comme un effet d'après coup, comme un utile anachronisme, en quelque sorte. Il insiste surtout sur l'alphabétisation qui élargit la culture commune et sur les types de lecture: lecture intensive et souvent communautaire de quelques textes presque sacrés contre lecture extensive et souvent individuelle de nombreux ouvrages de tous ordres. Dans ces relations tressées entre des formes matérielles, des dispositifs techniques, des usages culturels et des comportements de lecture, on a une façon complexe et originale de retracer

33. Jack Goody, La raison graphique. La domestication de la pensée sauvage [1977], trad. Jean Bazin et Alban Bensa, Paris, Les Éditions de Minuit, coll. «Le sens commun », 1979 .

34. Voir Elisabeth Lewisohn Eisenstein, La révolution de l'imprimé à l'aube de l'Europe moderne, trad. Maud Sissung et Marc Duchamp, Paris, La Découverte, 1991.

35. Roger Chartier, Culture écrite et société. L'ordre des livres: $14^{e}-18^{e}$ siècle, Paris, Albin Michel, 1996, p. 212. 
les origines culturelles de la Révolution ${ }^{36}$. On saisit sur cet exemple que les usages de lecture sont sans doute plus déterminants que les changements matériels de support, dont on tend parfois à réifier les effets. Bois, cire, argile, papyrus, parchemin, papier : tous ces changements importants de matériaux, qui ont aussi amené à concevoir de nouvelles formes de supports (tablette, rouleau, codex), sont ainsi moins déterminants que, par exemple, le déplacement de l'écriture cunéiforme, idéographique ou hiéroglyphique vers l'écriture alphabétique qui témoigne d'un bouleversement de la conception de l'écriture et de la posture mentale de lecture, car ce n'est plus comme en Mésopotamie, en Égypte ou en Chine, une façon de se raccorder au cosmos (même pour des instrumentations techniques ou des calculs utilitaires), c'est désormais une façon d'épouser le flux de la voix ${ }^{37}$. En même temps, on sort d'une maîtrise par une élite d'intermédiaires sacrés pour ouvrir l'écrit à n'importe qui, comme s'en plaint encore Platon dans le Phèdre.

Le système discursif - À partir de cette matérialité du médium, Friedrich Kittler en généralise le concept pour y inclure toute forme de communication dans un contexte historique donné: poésie ou théâtre sont des composantes de ces médialités, comme autant de moyens de création, stockage et diffusion de données ${ }^{38}$. Comme toute forme de transmission, les médias nécessitent des canaux matériels et techniques qui font circuler non seulement des informations mais aussi des bruits ou des parasites. C'est à la surface des messages que réside tout leur sens et non dans les profondeurs opaques du passé ou de la vérité: la question de la signification est alors dénouée de toute quête herméneutique. La notion même de «société » apparaît comme un artifice conceptuel ou une justification insoupçonnée d'un statu quo politique. Ces médialités édifient des sous-systèmes de communication: ainsi les inventions et les diffusions autour de 1900 de la machine à écrire, du gramophone et du film font apparaître de manière inattendue le langage lui-même comme moyen de communication au moment où l'écrit ne constitue plus le seul moyen technique de stockage des données.

C'est justement la confrontation de ces technologies de médiation (et non une seule) qui change le rapport aux échanges linguistiques ou aux constructions intellectuelles. Friedrich Kittler peut ainsi voir les trois dimensions de la

36. Roger Chartier, Les origines culturelles de la Révolution française, Paris, Éditions du Seuil, coll. «L'univers historique», 1990.

37. Voir Henri-Jean Martin, Histoire et pouvoirs de l'écrit, Paris, Librairie académique Perrin, 1988, ainsi qu'Anne-Marie Christin (dir.), Histoire de l'écriture. De l'idéogramme au multimédia, Paris, Flammarion, 2001.

38. Friedrich A. Kittler, Aufschreibesysteme 1800-1900, Münich, Fink, 1995. 
construction de soi et des autres chez Lacan comme autant de figures médiales: l'écrit implique les différences de l'ordre symbolique, le cinéma transmet des contenus imaginaires, le phonographe enregistre le réel. Cela signifie que l'on ne traduit (au sens d'un équivalent général) pas un média dans un autre, on doit transposer en acceptant les inadéquations, les sauts, voire les violences: l'interprétation n'est qu'une instance de la technique générale de transposition des médias, comme l'exemplifie Freud, dans ce tournant de 1900, en créant cette interface entre le «script pictographique» des rêves et leurs contenus de pensée réorganisés.

Ces éléments ne constituent pas seulement des modifications de l'espace intellectuel; ils modifient les pratiques sociales: ainsi, la machine à écrire permet aux femmes, auxquelles le système d'enseignement n'est guère ouvert, de remplacer rapidement les hommes aux postes de secrétaires, tout en les reconfigurant comme un prolongement de la machine. Opportunité sociale et oppression symbolique sont également des productions médiales. Cela donnera l'occasion à Billie Wilder dans Ready, Willing, and Able (1937) de faire danser les girls sur le clavier géant d'une machine à écrire, comme les doigts experts des secrétaires dansent sur les touches, ou à la figure érotique de la secrétaire de prendre le tour du harcèlement sexuel. Bref, un «système discursif» désigne le réseau de technologies et d'institutions qui permet à une culture donnée de sélectionner, conserver et transmettre des données jugées pertinentes. Ces réseaux d'information ne peuvent être décrits qu'en contraste les uns avec les autres. On voit ici opérer une conception saussurienne, voire structurale, où la langue ne signifie qu'à partir des différences phonétiques et sémantiques: de même, les sous-systèmes médiatiques font sens par leurs différences intrinsèques. Autre manière de mobiliser encore la différence comme principe.

\section{AGIR}

Les dispositifs - La notion de «dispositif» est souvent reprise aujourd'hui, popularisée sans doute par l'usage qu'en fait Michel Foucault dans son Histoire de la sexualité (1976-1984). Alors qu'il parlait plutôt dans ses précédents travaux d'épistémè comme d'un champ de savoir cohérent qui s'imposait à une certaine époque, il recourt au concept de dispositif afin de proposer des analyses plus circonscrites et surtout moins hégémoniques. Le dispositif est un ensemble d'éléments hétérogènes discursifs ou non discursifs (institutions, discours, lois, énoncés scientifiques, etc.) qui règle la production de ce qui peut être considéré comme connaissable ou déchiffrable. Ainsi, le dispositif de sexualité ne concerne 
pas tant l'obsession ou la répression du sexe que sa mise en scène possible, voire son invention. Au dispositif juridique de l'alliance qui règle les lignages s'adjoint (en le contestant) un dispositif de sexualité qui régule des techniques mobiles de pouvoir. C'est que l'on est passé d'un pouvoir qui capture et prélève (des richesses, des biens, du travail) à un pouvoir qui produit et contrôle (des populations, des vies, des corps). Il ne faut donc pas placer «le sexe du côté du réel, et la sexualité du côté des idées confuses et des illusions; la sexualité est une figure historique très réelle, et c'est elle qui a suscité comme élément spéculatif, nécessaire à son fonctionnement, la notion de sexe ${ }^{39} »$.

Le dispositif de sexualité désigne ainsi cette mécanique des rapports de pouvoir qui tresse certaines relations entre les êtres, les choses et les idées. «C'est le dispositif qui permet de séparer, non pas le vrai du faux, mais l'inqualifiable scientifiquement du qualifiable ${ }^{40}$. » Le dispositif est ainsi toujours inscrit dans un rapport de pouvoir, producteur de formes de savoir, mais aussi conditionné par elles. Dans ce circuit entre pouvoir et savoir, l’important réside dans le « réseau qu'on peut établir entre tous ces éléments ${ }^{41}$ » ainsi façonné et analysable.

À la suite de Michel Foucault, Giorgio Agamben considère que même les objets de consommation courants peuvent devenir des dispositifs structurants : la télévision, l’ordinateur, le téléphone portable ont modifié en partie nos habitudes, mais aussi nos subjectivités, nos manières d'être au monde et avec les autres. Pour lui, un dispositif est:

tout ce qui a, d'une manière ou d'une autre, la capacité de capturer, d'orienter, de déterminer, d'intercepter, de modeler, de contrôler et d'assurer les gestes, les conduites, les opinions et les discours des êtres vivants. Pas seulement les prisons donc, les asiles, les écoles [...], les usines, les disciplines, les mesures juridiques, dont l'articulation avec le pouvoir est en un sens évidente, mais aussi le stylo, l'écriture, la littérature, la philosophie, l'agriculture, la cigarette [...] et pourquoi pas le langage lui-même ${ }^{42}$.

C'est dire qu'ils font partie du processus même d'hominisation : on pourrait ici encore reprendre l'idée d'André Leroi-Gourhan faisant de la station debout et

39. Michel Foucault, Histoire de la sexualité, 1. La volonté de savoir, Paris, Gallimard, coll. «Bibliothèque des histoires", 1976, p. 207.

40. Michel Foucault, «Le jeu de Michel Foucault», Ornicar, n 10, juillet 1977, repris dans Dits et écrits, III, Paris, Gallimard, coll. «Bibliothèque des sciences humaines », 1994, p. 301.

41. Ibid., p. 299.

42. Giorgio Agamben, Qu'est-ce qu'un dispositif?, trad. Martin Rueff, Paris, Éditions Payot \& Rivages, 2007, p. 31. 
de la mémoire collective autant d'étapes cruciales de ce processus. Ce seraient là les premiers dispositifs.

Giorgio Agamben distingue, d'un côté, les êtres vivants (les substances), de l'autre, les dispositifs. Entre eux, dans leur relation sans cesse mouvante: les sujets. En quelque sorte, sur la matière du vivant, le dispositif impose une forme: celle de tel ou tel sujet, c'est une "machine qui produit des subjectivations ${ }^{43}$ ». Une même substance peut ainsi recevoir diverses subjectivations: automobiliste, amoureux, alcoolique, avocat, ainsi de suite. Cependant, cette production est aussi une manière de mettre à distance de la vie les individus singuliers. C'est une forme de domination productive qu'il faudrait tâcher de profaner (c'est-à-dire de remettre dans la circulation commune des existences, des «appropriations", pour reprendre un terme déjà exploité). On ne peut, en effet, s'extasier simplement sur l'intérêt des réseaux et la saveur des relations sans saisir les rapports de force qui s'y glissent et les structures de pouvoir qu'ils impliquent.

Les appareils - La notion d'appareil mise de l'avant par Jean-Louis Déotte est souvent liée à la production des apparences ou à des formes d'apparaître, donc à des jeux de surface. Mais il faut prendre pour lui-même ce qu'il souligne chez Benjamin : «l'interprétation (herméneutique) est sans valeur pour lui. [...] Ce qui nous conduira à dire que pour lui la photo et le cinéma sont des appareils de vérité plus que des dispositifs de perception ${ }^{44}$. » Si l'appareil a affaire avec l'apparaître, ce n'est pas sous le signe phénoménologique de la perception ni sous celui, herméneutique, de l'interprétation : l'appareil fait apparaître, il ne dispose pas simplement à recevoir ou même à enregistrer.

Lappareil est la médiation entre trois instances: le corps, la loi et l'événement. «L'appareil, c'est donc la médiation entre le corps (la sensibilité affectée) et la loi (la forme vide universelle) »; «Un appareil est un certain mode d'enchaînement légal sur l'événement ${ }^{45}$.» Loin d'être circonscrit abusivement par l'ordre juridique, c'est par la loi que tout corps peut être ouvert à l'événement. En rendant "pareils» des corps (ce qui n'équivaut pas à les rendre tous identiques), l'appareil les ouvre à la forme universelle de la loi quand elle prend consistance dans un événement.

On peut retrouver cette même puissance positive dans une autre caractéristique de l'appareil : le fait qu'il ne tourne pas à l'exercice d'une domination, mais

43. Ibid., p. 42.

44. Jean-Louis Déotte, L'époque des appareils, Paris, Éditions Lignes-Manifeste, 2004, p. 211-212.

45. Ibid., respectivement p. 28 et p. 103. 
qu'il ouvre plutôt sur une émancipation, même si les appareils nécessitent un passage par l'imaginaire, et même si certains d'entre eux sont bien des institutions culturelles d'État (les musées par exemple), il n’est pas question de ramener les appareils à un ordre étatique ou à une instance de domination : «la spécificité des appareils est de nous émanciper de l'adhésion originaire au corps et aux lieux. Les appareils suspendent, déracinent, arrachent, délocalisent, déplacent violemment les $\operatorname{corps}^{46}$. » Ainsi, le cinéma devient appareil à partir du moment où il repose sur le montage, soit chez Eisenstein et Vertov. Car l'écriture par montage (Kracauer le disait aussi) respecte les conditions de la production industrielle tout en subvertissant le dispositif de production de la fantasmagorie. Cependant, « cet arrachement n'est jamais définitif, la continuité de la fantasmagorie reprend toujours le dessus, les appareils se mettent à réalimenter, selon leur régime propre, la rêverie de l'intérieur ${ }^{47}$ »; autrement dit, le dispositif récupère la puissance d'un appareil pour lui faire produire du continu, alors qu'un appareil faisait apparaître justement parce qu'il interrompait.

Cet exemple est significatif de la place allouée à l'art. En effet, même si les appareils donnés en exemple ne concernent pas tous le domaine artistique (pour la modernité : la perspective, la camera obscura, le musée, la photographie, le passage urbain, le cinéma, la cure analytique, etc.) et même si «la situation d'un art à un moment quelconque n'est intelligible que si l'on ne confond pas tel art et tel appareil, par exemple la peinture et la perspective ${ }^{48}$ ", il n'en demeure pas moins qu'il semble que la vertu de l'interruption, de la subversion, de la discontinuité soit bien une effectivité des œuvres d'art. À la différence du dispositif, l'appareil introduit de nouvelles formes de temporalité, quelque chose à quoi nous ne sommes pas par avance disposés. C'est pourquoi la question de la discontinuité a autant d'importance, parce qu'elle implique de rompre avec une conception de la temporalité en en proposant une autre expérience. L'appareil pose à chaque fois le problème de sa propre «époqualité »: comment faire époque? Autrement dit, comment organiser la venue (toujours surprenante et inattendue) de l'événement? - d'où le titre même de l'ouvrage: L'époque des appareils.

46. Ibid, p. 52 .

47. Ibid, p. 106.

48. Ibid., p. 53 . 


\section{RELIER}

Médiologie et médiacultures - On discerne sans doute la proximité des analyses intermédiales avec les travaux inspirés par la médiologie de Régis Debray, pour qui «l'objet de l'étude n'est pas un objet, ni une région du réel (les médias), mais les rapports entre objets, ou régions » car «le milieu extérieur est intérieur au message ${ }^{49}$ ». Cependant, il s'agit surtout de saisir comment un milieu technologique ordonne, de l'intérieur, les interventions humaines. Le risque est de verser dans un déterminisme rapide, qui fait l'économie de la complexité des situations :

L’outillage d'une société, qui constitue pour ainsi dire l'ossature de ses chairs symboliques (de sa mentalité, de son imaginaire, ses croyances, son rapport concret à l'espace et au temps abstrait), détermine un espace-temps pratique, mesuré par ses capacités (techniquement déterminées) de mise en mémoire et de déplacement ${ }^{50}$.

Le choix éloquent de la métonymie: squelette/technique et chair/symbolique donne à la discipline nouvelle de la médiologie sa colonne vertébrale...

Le «système dominant de conservation des traces (saisie, stockage et circulation) sert de noyau organisateur à la médiasphère d'une époque donnée » et devient, comme l'était l'infrastructure économique pour Marx, une détermination de la superstructure symbolique, au point que «chaque médiasphère suscite

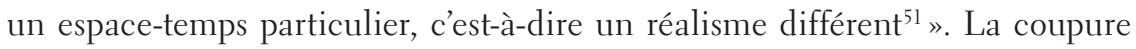
temporelle entre passé et présent instaurée par la modernité est ainsi rejouée pour les médiasphères. Régis Debray en distingue trois : logosphère (dominée par l'écriture), graphospère (dominée par l'imprimerie), vidéosphère (dominée par l'audiovisuel). Louise Merzeau en ajoute une quatrième récente, l'hypersphère $(\text { dominée par les réseaux })^{52}$.

La médiologie ouvre sur une science des médiations, dont le fréquent souci du détail révélateur n'a d'égal que les vastes classements aux synthèses rapides. D’autre part, héritant moins de l'histoire de l'imprimé que de l'histoire

49. Régis Debray, «Histoire des $4 M$ [message, médium, milieu, médiation] », Les cahiers de médiologie, n 6, «Pourquoi des médiologues? », 1998, p. 23.

50. Ibid., p. 20.

51. Régis Debray, Cours de médiologie générale, Paris, Gallimard, coll. «Bibliothèque des idées ", 1991, p. 313-314.

52. Pour le tableau des trois sphères qui recense toutes les formes sociales des médiations depuis l'invention de l'écriture: ibid., p. 334-335. Pour l'hypersphère, voir Louise Merzeau, "Ceci ne tuera pas cela», Les cahiers de médiologie, n 6, "Pourquoi des médiologues », 1998, p. 36-37. 
des mentalités (tout en produisant une archéologie de la vitesse plutôt qu'une histoire de la lenteur), et plus généralement d'un modèle herméneutique, la médiologie traque sous les effets de surface l'être caché des profondeurs ${ }^{53}$. Enfin, la médiologie cherche des formes de détermination par la technique des imaginaires singuliers ou des symboliques collectives, et moins des systèmes de co-construction entre supports techniques, autorités institutionnelles, pratiques sociales et configurations contingentes.

On peut, certes, suivre les variations techniques en fonction des contextes culturels et institutionnels, comme le propose Daniel Bougnoux, par exemple avec l'imprimerie: «En Chine, l'écriture idéographique réclame d'énormes capitaux, c'est une affaire d'État dont le développement renforce en retour le pouvoir central ; en Occident, l'invention de la presse à caractères mobiles déconcentre et libère la circulation du livre, démocratisant du même coup l'accès au savoir ${ }^{54}$.» Cependant, en Chine, la calligraphie manuscrite demeure un privilège des mandarins, constituant une classe d'élite gardant le monopole de l'administration étatique, alors que les régimes royaux de censure et de privilège aux libraires participent de la construction des États modernes occidentaux. Il faut donc allier enquête institutionnelle et formes de subjectivation aux innovations technologiques. Comme nous l'avons vu, on a longtemps considéré, avec exagération, que la Révolution française était issue des critiques (aux implications démocratiques) des philosophes des Lumières. Ce causalisme des idées sur les événements peut toujours être renversé en causalisme de la technique sur les idées, il n'en est pas plus juste.

Les sociologues de la communication ont tâché, eux aussi, de penser les formes sociales de subjectivation en liaison avec les changements techniques et les évolutions institutionnelles. La communication ne passe pas seulement par la voix ou l'écrit, par la télévision et le téléphone. Il faut encore des médias de transmission dont un des plus fondamentaux est les voies de communication: chemins de terre, routes pavées, fleuves ou canaux ${ }^{55}$. Ensuite, peuvent se mettre en place des formes économiques et instituées qui en exploitent les possibilités comme la messagerie royale en France au $17^{\mathrm{e}}$ siècle ou le Poney Express aux États-Unis au

53. «La télédétection par satellite sait à présent établir une corrélation entre le mouvement des vagues en surface et le relief des fonds océaniques. Ainsi le médiologue détecte par la surface les profondeurs d'un courant de pensée, d'une famille d'esprit, d'une mouvance.» Debray, 1991, p. 78.

54. Daniel Bougnoux, La communication par la bande. Une introduction aux sciences de l'information et de la communication, Paris, La Découverte, 1998, p. 43.

55. Armand Mattelart, L'invention de la communication, Paris, La Découverte, 1994. 
$19^{\mathrm{e}}$ siècle. Cependant certains développements techniques suscitent plus que des aménagements sociaux, ils provoquent des déplacements culturels, comme on le voit avec la «culture de l'imprimé». Ainsi, apparaissent ce que l'on peut appeler des «médiacultures». Intégrant les développements des cultural studies et des études postcoloniales, des sociologues comme Éric Macé et Éric Maigret tâchent ainsi de donner aux analyses des médias une profondeur de champ sociologique et idéologique conséquente ${ }^{56}$. L'intérêt est ici d'étendre une étude technique ou même sociale de tel ou tel média à une mise en relation de tous les éléments qui constituent un champ culturel (au sens anthropologique du terme).

Le médium du mineur: New Historicism et microhistoire, études féministes et postcoloniales - Autant la médiologie semble, au moins chez Régis Debray, adepte des grandes synthèses, elle n'en met pas moins sous tension le détail et l'anodin. Reprenant peut-être un souci particulier de la déconstruction derridienne qui cherche volontiers dans un motif anecdotique une façon de défaire la tapisserie conceptuelle des textes, elle se rapproche d'une manière caractéristique du New Historicism qui cherche à construire des tableaux d'histoire à partir de petits événements ${ }^{57}$ ou de la microhistoire ${ }^{58}$, qui tente de lire dans des formes d'appropriation singulière les façons de vivre réellement les grands processus de l'histoire. L'important consiste en fait à demeurer au plus proche de l'anecdote ${ }^{59}$ et du détail, au plus près des acteurs eux-mêmes et des processus d'écriture, pour mieux y apercevoir les singularités, les reliefs spécifiques, les manières d'aménager ou de s'approprier les grands éléments d'une culture. On trouve ainsi plus d'intérêt à suivre les évolutions variées du surfeur plutôt que la vague inéluctable qui le porte. Cela donne une place au mineur ${ }^{60}$ et au détail dans lesquels

56. Éric Macé et Éric Maigret (dir.), Penser les médiacultures. Nouvelles pratiques et nouvelles approches de la représentation du monde, Paris, Armand Colin, 2005.

57. Voir Stephen Greenblatt et Catherine Gallagher, Practising New Historicism, Chicago, The University of Chicago Press, 2001.

58. Voir Carlo Ginzburg, Le fromage et les vers: l'univers d'un meunier du $16^{e}$ siècle, trad. Monique Aymard, Paris, Flammarion, coll. «Nouvelle bibliothèque scientifique », 1980.

59. Voir Joel Fineman, «The History of the Anecdote: Fiction and Fiction », dans Harold Aram Veeser (dir.), The New Historicism, New York et Londres, Routledge, 1989; Marie-Pascale Huglo, Métamorphoses de l'insignifiant: essai sur l'anecdote dans la modernité, Montréal, Balzac-Le Griot éditeur, 1997; ainsi que Jane Gallop, Anecdotal Theory, Durham, Duke University Press, 2002.

6o. Voir Gilles Deleuze et Félix Guattari, Kafka. Pour une littérature mineure, Paris, Les Éditions de Minuit, coll. «Critique», 1975. 
se réfractent enjeux de pouvoir et formes de domination, ainsi que les études féministes et postcoloniales se sont attachées à en reconfigurer les effets. Naomi Schor met d'ailleurs en relief le fait que la place allouée aux détails rejoue la position secondaire des femmes ${ }^{61}$ : à la place publique des grandes affaires politiques répondent les minuscules tâches quotidiennes du domestique.

Même si l'histoire quantitative et sérielle a son importance, on voit dans ces procédures l'intérêt de penser par cas précis plutôt que par brassées d'ensemble. La critique du canon visait la constitution historique d'une sélection d'exemples qui privait de visibilité des pratiques culturelles que la mondialisation et l'attention aux processus de minorisation ont rendue plus perceptible grâce aux études féministes et postcoloniales. Pourtant, ce souci du mineur n'a pas débouché sur de nouvelles pratiques de lecture du petit et du détail, malgré la présence très forte de la figure de Walter Benjamin dans les études culturelles. En effet, l'image dialectique ou la dialectique à l'arrêt ${ }^{62}$ sont autant de figures qui valorisent justement le fait de partir d'un événement bloqué, d'une forme d'exemplarité.

On peut rapprocher cette construction de l'exemplarité de ce que reconnait, dans une perspective de sociologie historique, Claude Imbert comme étude de cas:

Une étude de cas projette, sur une réalité qu'il ne s'agit plus de dissoudre dans le cours des faits - c'est-à-dire selon une épistémologie par défaut -, un espace virtuel d'intelligibilité. Elle n'ignore pas la courbure qu'y impriment des figures symboliques et contractuelles instables, et pourtant essentielles parce qu'elles portent la réalité d'un processus de socialité où des capacités anthropologiques et cognitives composent avec des contraintes et des commodités techniques, éminemment composites et révisables ${ }^{63}$.

Le geste exemplaire constitue bien une projection - donc une lecture -, à la fois projection hors de son temps propre, hors de la singularité de son contexte, et projection sur le monde des événements pour mieux leur donner la virtualité d'une réitération, autrement dit d'une valeur et d'un sens. Ainsi devient-il indispensable de penser les manières de relier moins le particulier et le général que le minuscule du détail et l'intensité du sens.

61. Naomi Schor, Reading in Detail: Aesthetics and the Feminine, New York, Routledge, 1989.

62. Walter Benjamin, Paris, capitale du $19^{e}$ siècle: le livre des passages, trad. Jean Lacoste, Paris, Éditions du Cerf, 1993.

63. Claude Imbert, «Le cadastre des savoirs. Figures de connaissance et prises de réel », dans Jean-Claude Passeron et Jacques Revel (dir.), Penser par cas, Paris, Éditions de l'ÉHESS, 2005, p. 279. 
Hypertextualité - Aristote faisait du paradigme une des preuves de la rhétorique et une pratique de l'induction où «l'exemple ne présente les relations ni de la partie au tout, ni du tout à la partie, mais seulement de la partie à la partie, du semblable au semblable, lorsque les deux termes rentrent dans un même genre mais que l'un est plus connu que l'autre ${ }^{64}{ }^{\text {}}$. Cette manière de lier une partie à une autre partie constitue une opération que nous menons quotidiennement grâce au développement de l'informatique: les hyperliens. Ceux-ci ne constituent pas de système hiérarchisé, où l'on irait paisiblement du petit au grand, du singulier à l'universel, du multiple à l'Un. Au contraire, ils déploient grâce à l'inscription des programmeurs et à la performance des usagers, des parcours variables, ouverts, non linéaires.

Ainsi, nous revenons en boucle aux premiers éléments que nous notions. En effet, l'hypertextualité est proche des pratiques du dialogisme et de l'intertextualité. On peut même noter un effet de contemporanéité, étant donné que, à l'instar du concept d'intertextualité, le terme apparaît au milieu des années 1960 chez Doug Engelbart et se développe surtout dans les décennies qui suivent. Pourtant, on a souvent opposé la linéarité supposée des textes imprimés, ainsi que leur caractère statique, au potentiel dynamique et ouvert de l'hypertexte. La prise en compte de l'intertextualité a au moins montré que cette opposition ne tient pas et que l'on peut même penser avec profit la lecture de l'imprimé à partir de l'hypertexte, comme un processus ouvert où le lecteur s'approprie l'œuvre. Cependant, l'hypertexte permet de déployer plus systématiquement cette absence de système centré sur une intention première qu'il faudrait découvrir. Comme le résume bien Jerome McGann à propos de l'hypertexte: «One is encouraged not so much to find as to make order - and then to make it again and again ${ }^{65}$.»

L'hypertexte propose des liens paradigmatiques, laissant le parcours syntagmatique aux choix ponctuels de ses lecteurs ${ }^{66}$. Mais c'est là sans doute où se glisse la présence de l'appareillage technique et ses effets de retour sur la culture de l'imprimé: «digital document, because its perceptible form is always being manufactured just-in-time, on the spot, can't ever sever its relationship to a set

64. Aristote, Rhétorique, trad. Médéric Dufour, Paris, Les Belles Lettres, 1967, I, 1357 b 26 .

65. Jerome John McGann, Radiant Textuality: Literature after the World Wide Web, New York, Palgrave, 2001, p. 71.

66. Voir David Piotrowski, L'hypertextualité ou la pratique formelle du sens, Paris, Champion, 2004. 
of manufacturing technologies ${ }^{67}$ ». Cet effet de variation et de présence ramène l'hypertexte à une technologie de la voix plus encore que de l'imprimé - ce qui nous fait sentir la nécessité de sortir d'une conception puriste des médias. Chaque médium ne délimite pas son territoire de façon ordonnée et jalouse. S'il existe bien des conflits entre médias, chacun empiète sur les usages du voisin, récupère l'énergie du concurrent, rejoue la séduction de son partenaire. Jay Bolter l'indique: nous avons là autant de processus de remédiation ${ }^{68}$.

Bien sûr, il ne faut pas considérer qu'avec le développement de ces hyperliens, nous serions projetés dans un cyberespace immatériel. Les circuits de silicium sur lesquels nos documents virtuels sont inscrits grâce à des flux d'électrons sont bien matériels, même si ce sont des états de la matière que nous modifions sans cesse. C'est pourquoi la question de la matérialité spécifique des médias continue à importer. Katherine Hayles a critiqué la vision de la littérature indépendante de ses formes matérielles et de ses usages de lecture comme si l'on prenait celles-ci pour des accidents opposés à l'essence de l'œuvre: «print literature was widely regarded as not having a body, only a speaking $\operatorname{mind}^{69}{ }^{\text {». Il }}$ en va de même pour les médias numériques. Matérialité et technique doivent de nouveau être mobilisées afin de saisir leur interaction avec les formes culturelles, les effets institutionnels, le monde intellectuel et les outillages mentaux. C'est ce qui amène à se retourner en définitive sur cet univers de l'intellect:

On peut bien admettre avec Ted Nelson ou Jay Bolter que l'hypertexte permet de relier entre elles des idées élémentaires, mais la question est de déterminer en quoi consiste une idée. Comment segmenter la coulée du texte? À partir de quel moment convient-il de faire migrer l'amorce d'un développement nouveau vers une autre page, de faire apparaître un autre rhizome dans une structure proliférante, d'établir un lien avec un autre fragment? Rédiger un hypertexte amène ainsi à s'interroger inlassablement sur la notion même de texte ou de fragment ${ }^{70}$.

Voire à s'interroger sur l'idée même d'idée et rouvrir la question métaphysique de l'origine des idées ${ }^{71}$.

67. David M. Levy, Scrolling Forward. Making Sense of Documents in the Digital Age, New York, Arcade Publishing, 2001, p. 152.

68. Jay David Bolter, Writing Space: Computers, Hypertext, and the Remediation of Print, Mahwah, Lawrence Erlbaum Associates, 2001.

69. Nancy Katherine Hayles, Writing Machines, Cambridge (Mass.), MIT Press, 2002, p. 32.

70. Christian Vandendorpe, Du papyrus à l'hypertexte. Essai sur les mutations du texte et de la lecture, Montréal, Boréal, 1999, p. 244.

71. Je me permets de renvoyer à mon ouvrage D'où nous viennent nos idées? Métaphysique et intermédialité, Montréal, VLB Éditeur, 2010. 
Pour clore ce parcours, on peut remarquer que les dispositifs techniques de l'hypertexte de même qu'Internet ont été inventés et développés pour répondre au risque de coupure des communications vers un centre de décision en cas de conflit majeur. De même, la construction exemplaire, à partir des lectures de cas particuliers et de détails significatifs, est un lieu de tensions, de conflits, un champ de forces, un espace où s'entremêlent les désirs de domination ou les appétits de soumission des uns et des autres. Il n'est peut-être pas indifférent que le verbe epagô, utilisé par Aristote et que l'on traduit par le terme technique d'«induire», désigne avant tout le fait d'amener des vivres ou des alliés pour un combat, de marcher à l'ennemi, d'asséner un coup ou d'intenter un procès. Quand Cicéron rend le nom epagôgé par inductio, il exploite le même réseau de sens que le grec, avec la signification supplémentaire de «recouvrir» un bouclier de peau ou un corps d'une tunique, d'où l'idée de voiler, de tromper, d'induire en erreur, voire de représenter et de mettre en scène. En grec comme en latin, l'«induction » tient autant à la puissance qu'au charme.

Linduction exemplaire n'est donc pas la disposition neutre d'un espace logique; c'est un dispositif conflictuel qui préside à l'arrangement des forces en présence pour une meilleure pénétration, on pourrait presque dire une plus grande séduction des événements. «Deux visages semblables, dont aucun ne fait rire en particulier, font rire ensemble par leur ressemblance », affirmait Pascal. On pourrait dire la même chose de deux (ou plusieurs) conceptions théoriques. Que l'on soit secoué par le comique des ressemblances inattendues ou par l'illumination de rapprochements occasionnels, on y aura gagné une nouvelle manière de regarder les idées et les individus, les théories et leurs histoires, à partir d'un certain point de vue. Car un choix, bien sûr, a présidé à ces exemples: chaque famille a ses personnages préférés, ses oncles d'Amérique et ses fantômes dans le placard. Nous voyons la ressemblance là où nous la désirons, là où elle nous touche.

Il est possible d'opérer ainsi pour toutes sortes de théories. Cependant, le creuset de ressemblances que l'intermédialité occupe montre combien la pratique des juxtapositions imprévues et des rapprochements conflictuels lui est inhérente. Issue de pensées de la différence, peut-être trouve-t-elle plus encore de validité à réfléchir les ressemblances comme autant de mises en relation. L'important, comme dans toute réunion de famille, est que ça se passe mal, mais qu'on se retrouve l'année suivante. 